\title{
Analytical and Approximate Solutions of a Novel Nervous Stomach Mathematical Model
}

\author{
Yolanda Guerrero Sánchez $\mathbb{D}^{1},{ }^{1}$ Zulqurnain Sabir, ${ }^{2}$ Hatıra Günerhan, ${ }^{3}$ \\ and Haci Mehmet Baskonus $\mathbb{D}^{4}$ \\ ${ }^{1}$ Universidad de Murcia, Departamento de Anatomía y Psicobiología, 30001 Murcia, Spain \\ ${ }^{2}$ Department of Mathematics and Statistics, Hazara University, Mansehra, Pakistan \\ ${ }^{3}$ Mathematics Department, Faculty of Education, Kafkas University, Kars, Turkey \\ ${ }^{4}$ Faculty of Education, Harran University, Sanliurfa, Turkey \\ Correspondence should be addressed to Yolanda Guerrero Sánchez; yolanda.guerreros@um.es
}

Received 20 April 2020; Accepted 8 June 2020; Published 4 July 2020

Academic Editor: Jia-Bao Liu

Copyright () 2020 Yolanda Guerrero Sánchez et al. This is an open access article distributed under the Creative Commons Attribution License, which permits unrestricted use, distribution, and reproduction in any medium, provided the original work is properly cited.

\begin{abstract}
The stomach is usually considered as a hollow muscular sac, which initiates the second segment of digestion. It is the most sophisticated endocrine structure having unique biochemistry, physiology, microbiology, and immunology. The pivotal aim of the present study is to propose the nonlinear mathematical model of the nervous stomach system based on three compartments namely, tension $(T)$, food $(F)$, and medicine $(M)$. The detailed description of each compartment is provided along with the mathematical form and different rates/factors, such as sleep factor, food rate, tension rate, medicine term, and death rate. The solution of the designed model is presented numerically by using the well-known differential transformation technique. The behavior of the obtained solution has been captured with respect to time as well as presentations of the numerical simulations.
\end{abstract}

\section{Introduction}

The importance of every organ in the human body has its individual value, and without having any organ or having a weak organ, the human body feels troubles. In human body, every organ is linked to other organs, e.g., eye has a connection with the nose and the nose is connected with the ear. The stomach is one of the most significant organ in the human body that has connection with many organs. The stomach's role is maintaining the health of every living being, and it magnetizes the attention of the research community [1]. The earliest Greeks observed the gastric contents with the bitter nature. In the sixteenth century, both van Helmont [2] and Paracelsus [3] believed that an acid is present in the stomach, and it is essential for digestion. Consequent observations by Spallanzani [4] and De Reaumur [5] recommended the 'solvent' properties of digestive juice on the tissue of animals. Prout [6] did not publish his work related to the gastric acid secretion nature until 1823. Beaumont [7] provided the first interpretations on his patient with gastric fistula, and that was published by Alexis St Martin three years later in the second decade of the eighteenth century. These observations based on meticulous were discussed for about a hundred years, and the core of his investigations was gastric ingestion in a human body with the stress effects.

At the beginning of the twentieth century, the control of gastric secretion was discovered by ablation of the coeliac axis and vagotomy as therapeutic interferences. This highlighted the complex nature of the gastric secretion's control, and it led to a quick increase in interest and the work of Laidlaw and Dale on histamine [8], which led to the serious discovery by Popielski on the effects of histamine on gastric secretion [9]. Bayliss and Starling presented the secretin [10] 
and Edkins dissertation on gastrin [11]. These discoveries helped us to understand the gastric disease leading to dramatic advances in the pharmacological organization of peptic ulcer disease with the finding of the $\mathrm{H} 2$-receptor antagonists by Sir James Black in the seventh decade of the nineteenth century [12]. Marshall and Warren discussed the acid-related disease in the twentieth century until the groundbreaking discovery of Helicobacter pylori in 1983 [13]. The stomach was reflected microbiologically sterile, although Jaworski [14] has presented many observations of several bacterial populations in gastric juice.

The present study aims to illustrate the mathematical form of the nervous stomach model and the performance of the numerical solutions by considering all the parameters of the designed model via the differential transformation (DT) method. The considered novel technique is proposed by Zhou [15], called as a differential transformation method, with the help of the Taylor series expansion in the $8^{\text {th }}$ decade of the nineteenth century. The designed nervous stomach model is obtained by considering the three compartments namely, tension $(T)$, food $(F)$, and medicine $(M)$. Furthermore, for more convince, this model is named as TFM. Three different cases based on the designed model have been taken, and the numerical results are drawn using the DT method. The obtained results show the efficiency of the proposed scheme.

Some salient geographies of the designed TFM model are given as follows:

(i) The nervous stomach model is presented effectively by using the three compartments namely tension $(\mathrm{T})$, food $(F)$, and medicine $(M)$

(ii) The detailed descriptions of all the compartments of the TFM model are provided along with different factors and rates

(iii) The nonlinear system of ordinary differential equations is obtained by using the three abovementioned compartments

(iv) The numerical solutions of the designed TFM model are performed by using the DT numerical scheme

The rest of the paper is organized as follows. Section 2 describes the structure of the designed model. Section 3 presents the procedure of the DT scheme and essential results. Section 4 shows the detailed results and discussion of the TFM model. Conclusions along with future research directions are presented in the last section of the paper.

\section{Structure of the TFM Model}

The mathematical form of the nervous stomach model will be presented along with the three compartments namely tension $(T)$, food $(F)$, and medicine $(M)$. The details of each compartment are presented as follows: (i) $T(t)$ : the indications caused by mental health are always present in the mind. There are various physical symptoms; one of them that may target anybody and cause anxiousness and stress is a nervous stomach. The effect of a nervous stomach is extremely inconvenient for the human body and may forbid them from completing the projects due to stress. It is one of the main reasons to disturb the stomach at time $t$.

(ii) $F(t)$ : the oily, spicy, and crispy food are the main factors to disturb the stomach. Especially, anybody who does not frequently consume spicy foods and has no patience may cause havoc on the digestive system. The presence of some compounds in spicy food such as capsaicin is not broken down in digestion and can annoy the lining of the intestines or stomach. This is the main cause to disturb the stomach at time $t$.

(iii) $M(t)$ : the overuse of medicine is also an important factor to disturb the stomach. Most medicines are used to recover different disease that can also disturb the stomach. These medicines straightly upset the stomach, and through this way, the stomach feels irregularity and gets affected badly.

The mathematical form of the TFM model is presented in the nonlinear system of ordinary differential equations, which is achieved by using the three above compartments namely, tension, food, and medicine at time $t$, and it is defined as

$$
\begin{cases}\frac{\mathrm{d} T(t)}{\mathrm{d} t}=-\alpha F(t) T(t)-\beta, & T(0)=I_{1}, \\ \frac{\mathrm{d} F(t)}{\mathrm{d} t}=\delta T(t)+\gamma M(t) F(t), & F(0)=I_{2}, \\ \frac{\mathrm{d} M(t)}{\mathrm{d} t}=-\delta T(t)+\beta F(t) M(t)-d, & M(0)=I_{3},\end{cases}
$$

where $\alpha$ represents the quantity of food, and negative sign indicates the lesser quantity of food. $\beta$ shows the sleep factor, $\delta$ is used for the tension rate, $\gamma$ indicates the recovery term, and $d$ shows the death rate. Moreover, $I_{1}, I_{2}$, and $I_{3}$ are the initial conditions for all compartments of the TFM model. For the verification of the designed model, three different cases are considered based on the designed TFM model and solved by using a well-known DT method. The historical DT method is famous to solve the singular, nonlinear, and fractional differential models [16-23].

\section{Differential Transformation Scheme}

Taking one-dimensional arbitrary function $w(x)$ in Taylor's series about a point $x=x_{0}$, we have 


$$
W(k)=\frac{1}{k !}\left[\frac{\mathrm{d}^{k} w(x)}{\mathrm{d} x^{k}}\right]_{x=0} .
$$

The original function is $w(x)$ in the above equation (2), whereas the transformed function is $W(k)$, which is also known as T-function. The differential transform of the inverse of $W(k)$ is provided as follows:

$$
w(x)=\sum_{k=0}^{\infty} Y(k)\left(x-x_{0}\right)^{k} \equiv D^{-1} W(k) .
$$

Using equations (2) and (3) and taking $x_{0}=0$, the obtained form is written as follows:

$$
w(x)=\sum_{k=0}^{\infty} \frac{x^{k}}{k !}\left[\frac{d^{k} w(x)}{d x^{k}}\right]_{x=0} .
$$

Equation (4) gives the concepts of differential transform derived from the expansion of the Taylor series, and this scheme is not applied for symbolical evaluation of the derivatives. Moreover, comparative derivatives are found by using an iterative procedure. In the present study, the lower case letter is used to show the original function, and upper case letter is used to represent the transformed function. From the definition of the above two equations, one can easily prove that the transformed functions have the basic mathematics values provided in Table 1.

In real applications, $w(x)$ is obtained by a finite series, and equation (3) can be described as follows:

$$
w(x)=\sum_{k=0}^{m} x^{k} W(k) .
$$

Equation (5) shows that $w(x)=\sum_{k=m+1}^{\infty} x^{k} W(k)$ is very small and can be neglected. In fact, it denotes the error. Hence, in the present study, $m$ is used for the convergence of natural frequency.

Theorem 1. If $w(x)=a(x) b(x)$, then $W(k)=A(k) \otimes$ $B(k)=\sum_{l=0}^{k} A(l) B(k-l)$ (here $\otimes$ denotes the convolution):

$$
\begin{aligned}
w(x)= & \sum_{k=0}^{\infty} A(k)\left(x-x_{0}\right)^{k} \times \sum_{k=0}^{\infty} B(k)\left(x-x_{0}\right)^{k} \\
= & \left(A(0)+A(1)\left(x-x_{0}\right)+A(2)\left(x-x_{0}\right)^{2}+\ldots\right) \\
& \times\left(B(0)+B(1)\left(x-x_{0}\right)+B(2)\left(x-x_{0}\right)^{2}+\ldots\right) \\
= & A(0) B(0)+(A(0) B(1)+A(1) B(0))\left(x-x_{0}\right) \\
& +(A(0) B(2)+A(1) B(1)+A(2) B(0))\left(x-x_{0}\right)^{2}+\ldots \\
= & \sum_{k=0}^{\infty} \sum_{l=0}^{k} A(l) B(k-l)\left(x-x_{0}\right)^{k} .
\end{aligned}
$$

By using equation (3), we can write

$$
W(k)=\sum_{l=0}^{k} A(l) B(k-l)
$$

Theorem 2. If $w(x)=x^{m}$, then $W(k)=\delta(k-m)=$ $\left\{\begin{array}{ll}1, & k=m, \\ 0, & k \neq m,\end{array}\right.$ where $\delta(k)=\left\{\begin{array}{ll}1, & k=0, \\ 0, & k \neq 0,\end{array}\right.$.

Proof. By using equation (2), we have

$$
W(k)=\left.\frac{1}{k !} \frac{\partial\left(x^{m}\right)}{\partial x^{k}}\right|_{t=0}= \begin{cases}\frac{1}{k !} \frac{\partial^{k}\left(x^{k}\right)}{\partial x^{k}}=\frac{k !}{k !}=1, & k=m, \\ \frac{1}{k !} \frac{\partial^{k}\left(x^{m}\right)}{\partial x^{k}}=0, & k \neq m .\end{cases}
$$

Theorem 3. If $w(t)=u_{1}(t) u_{2}(t) u_{3}(t) \ldots u_{n-1}(t) u_{n}(t)$, then $W(k)=\sum_{l_{n-1}=0}^{k} \sum_{l_{n-2}=0}^{l_{n-1}} \cdots \sum_{l_{2}=0}^{l_{3}} \sum_{l_{1}=0}^{l_{2}} U_{1}\left(l_{1}\right) U_{2}\left(l_{2}-l_{1}\right) \cdots$ $U_{n-1}\left(l_{n-1}-l_{n-2}\right) U_{n}\left(k-l_{n-1}\right)$.

Proof. By using equation (2), we have 
TABLE 1: The essential operations of the unique and transformed functions of the DT method.

\begin{tabular}{lc}
\hline Unique function & Transformed function \\
\hline$w(x)=a(x) \pm b(x)$ & $W(k)=A(k) \pm B(k)$ \\
$w(x)=c a(x)$ & $W(k)=c A(k)$ \\
$w(x)=\left(\mathrm{d}^{m} a(x)\right) / \mathrm{d} x^{m}$ & $W(k)=(k+1)+(k+2) \cdots(k+m) \cdot A(k+m)$ \\
$w(x)=a(c x)$ & $W(k)=c^{k} \cdot A(k)$ \\
$w(x)=a(x / c)$ & $W(k)=A(k) / c^{k}$ \\
$w(x)=\left(\mathrm{d}^{m} / \mathrm{d} x^{m}\right) a(c x)$ & $W(k)=(k+1)+(k+2) \cdots(k+m) \cdot c^{k+m} \cdot A(k+m)$ \\
$w(x)=e^{x+c}$ & $W(k)=e^{c} / k !$ \\
\hline
\end{tabular}

$$
\begin{aligned}
W(0)= & \frac{1}{0 !}\left[u_{1}(t) u_{2}(t) \cdots u_{n-1}(t) u_{n}(t)\right]_{t=t_{0}} \\
= & U_{1}(0) U_{2}(0) \cdots U_{n-1}(0) U_{n}(0) \\
W(1)= & \frac{1}{1 !} \frac{\partial}{\partial t}\left[u_{1}(t) u_{2}(t) \cdots u_{n-1}(t) u_{n}(t)\right]_{t=t_{0}} \\
= & {\left[u_{1}^{\prime}(t) u_{2}(t) \cdots u_{n-1}(t) u_{n}(t)+u_{1}(t) u_{2}^{\prime}(t) \cdots u_{n-1}(t) u_{n}(t)+\cdots+u_{1}(t) u_{2}(t) \cdots u_{n-1}^{\prime}(t) u_{n}(t)\right.} \\
& \left.+u_{1}(t) u_{2}(t) \cdots u_{n-1}(t) u_{n}^{\prime}(t)\right]_{t=t_{0}} \\
= & U_{1}(1) U_{2}(0) \cdots U_{n-1}(0) U_{n}(0)+U_{1}(0) U_{2}(1) \cdots U_{n-1}(0) U_{n}(0)+\cdots+U_{1}(0) U_{2}(0) \cdots U_{n-1}(1) U_{n}(0) \\
& +U_{1}(0) U_{2}(0) \cdots U_{n-1}(0) U_{n}(1) \\
& +U_{1}(0) U_{2}(0) U_{3}(0) \cdots U_{n-2}(1) U_{n-1}(1) U_{n}(0)+U_{1}(0) U_{2}(0) U_{3}(0) \cdots U_{n-2}(0) U_{n-1}(1) U_{n}(1)
\end{aligned}
$$

We have

$$
\begin{aligned}
W(k)= & \sum_{l_{n-1}=0}^{k} \sum_{l_{n-2}=0}^{l_{n-1}} \cdots \sum_{l_{2}=0}^{l_{3}} \sum_{l_{1}=0}^{l_{2}} U_{1}\left(l_{1}\right) U_{2}\left(l_{2}-l_{1}\right) \\
& \cdots U_{n-1}\left(l_{n-1}-l_{n-2}\right) U_{n}\left(k-l_{n-1}\right) .
\end{aligned}
$$

$$
\begin{aligned}
k & =1: W(1)=\left.\frac{1}{1 !} \frac{\partial \sin (w t+\alpha)}{\partial t}\right|_{t=0}=\left.\frac{1}{1 !} w \cos (w t+\alpha)\right|_{t=0}=\left.\frac{1}{1 !} w \sin \left(\left(\frac{\pi}{2}+\alpha\right)+w t\right)\right|_{t=0}=\frac{1}{1 !} w \sin \left(\frac{\pi}{2}+\alpha\right) \\
k & =2: W(2)=\left.\frac{1}{2 !} \frac{\partial^{2} \sin (w t+\alpha)}{\partial t^{2}}\right|_{t=0}=\frac{1}{2 !} w^{2} \cos \frac{\pi}{2}+\left.(\alpha+w t)\right|_{t=0}=\left.\frac{1}{2 !} w^{2} \sin \left(\frac{\pi}{2}+\left(\alpha+\frac{\pi}{2}\right)+w t\right)\right|_{t=0} \\
& =\left.\frac{1}{2 !} w^{2} \sin \left(\frac{2 \pi}{2}+\alpha+w t\right)\right|_{t=0}=\frac{1}{2 !} w^{2} \sin \left(\frac{2 \pi}{2}+\alpha\right), \ldots, \\
k & =h: W(h)=\left.\frac{1}{h !} \frac{\partial^{h} \sin (w t+\alpha)}{\partial t^{h}}\right|_{t=0}=\left.\frac{w^{h}}{h !} \sin \left(\frac{h \pi}{2}+\alpha+w t\right)\right|_{t=0}=\frac{w^{h}}{h !} \sin \left(\frac{h \pi}{2}+\alpha\right)
\end{aligned}
$$

We have

$$
W(h)=\frac{w^{h}}{h !} \sin \left(\frac{h \pi}{2}+\alpha\right) .
$$

\section{Results and Discussion}

In this section, three different cases will be discussed by taking different values of the sleep factor, food rate, tension rate, medicine term, and death rate. These cases numerically 
TABLe 2: Numerical investigations of the $T(t), F(t)$, and $M(t)$ for Case 1 of the TFM model.

\begin{tabular}{|c|c|c|c|}
\hline \multirow{2}{*}{$t$} & \multicolumn{3}{|c|}{ Case 1} \\
\hline & $T(t)$ & $F(t)$ & $M(t)$ \\
\hline 0 & 0.9999 & 0.0001 & 0 \\
\hline 0.4 & 0.62731 & 0.67810 & -0.15969 \\
\hline 0.8 & -0.11289 & 0.87141 & -0.11953 \\
\hline 1.2 & -0.76466 & 0.47571 & -0.02268 \\
\hline 1.6 & -0.91441 & -0.24356 & 0.05255 \\
\hline 2 & -0.31034 & -0.72335 & -0.03076 \\
\hline 2.4 & 1.51535 & 0.02444 & -0.60601 \\
\hline 2.8 & 6.55651 & 4.51745 & -2.65702 \\
\hline 3.2 & 21.0587 & 20.2326 & -8.88120 \\
\hline 3.6 & 60.1631 & 67.4145 & -25.7791 \\
\hline 4 & 154.959 & 194.152 & -67.3316 \\
\hline 4.4 & 362.400 & 502.590 & -160.990 \\
\hline 4.8 & 778.453 & 1191.23 & -356.890 \\
\hline 5.2 & 1554.77 & 2619.56 & -741.403 \\
\hline 5.6 & 2919.070 & 5402.54 & -1456.36 \\
\hline 6 & 5199.07 & 10544 & -2725.58 \\
\hline 6.4 & 8850.05 & 19620 & -4890.42 \\
\hline 6.8 & 14485.3 & 35024.04 & -8456.69 \\
\hline 7.2 & 22909.1 & 60288.6 & -14155.1 \\
\hline 7.6 & 35150.96 & 100501 & -23018.7 \\
\hline 8 & 52500.24 & 162828 & -36478.7 \\
\hline 8.4 & 76539.23 & 257180 & -56485 \\
\hline 8.8 & 109172.9 & 397018 & -85652.1 \\
\hline 9.2 & 152653.1 & 600357 & -127437 \\
\hline 9.6 & 209593.8 & 890972 & -186354 \\
\hline 10 & 282975.4 & 129984 & -0.15969 \\
\hline
\end{tabular}

TABLE 3: Numerical investigations of the obtained results $T(t), F(t)$, and $M(t)$ for Case 2 of the TFM model.

\begin{tabular}{|c|c|c|c|}
\hline \multirow{2}{*}{$t$} & \multicolumn{3}{|c|}{ Case 2} \\
\hline & $T(t)$ & $F(t)$ & $M(t)$ \\
\hline 0 & 0.9999 & 0.0001 & 0 \\
\hline 0.4 & 0.99711 & 0.38141 & -0.16480 \\
\hline 0.8 & 1.14519 & 0.76386 & -0.35228 \\
\hline 1.2 & 1.45588 & 1.20409 & -0.58365 \\
\hline 1.6 & 1.96438 & 1.76437 & -0.88481 \\
\hline 2 & 2.73324 & 2.52173 & -1.28985 \\
\hline 2.4 & 3.85971 & 3.57910 & -1.84568 \\
\hline 2.8 & 5.48681 & 5.08036 & -2.61842 \\
\hline 3.2 & 7.81825 & 7.23190 & -3.70235 \\
\hline 3.6 & 11.1371 & 10.3342 & -5.23279 \\
\hline 4 & 15.8281 & 14.8287 & -7.40436 \\
\hline 4.4 & 22.4012 & 21.3651 & -10.4966 \\
\hline 4.8 & 31.5164 & 30.8991 & -14.9098 \\
\hline 5.2 & 44.0051 & 44.8295 & -21.2138 \\
\hline 5.6 & 60.8851 & 65.1876 & -30.2136 \\
\hline 6 & 83.3624 & 94.8947 & -43.0369 \\
\hline 6.4 & 112.814 & 138.106 & -61.2485 \\
\hline 6.8 & 150.746 & 200.663 & -86.9977 \\
\hline 7.2 & 198.705 & 290.679 & -123.207 \\
\hline 7.6 & 258.144 & 419.281 & -173.809 \\
\hline 8 & 330.223 & 601.560 & -244.041 \\
\hline 8.4 & 415.518 & 857.740 & -340.814 \\
\hline 8.8 & 513.633 & 1214.63 & -473.152 \\
\hline 9.2 & 622.681 & 1707.40 & -652.739 \\
\hline 9.6 & 738.609 & 2381.74 & -894.564 \\
\hline 10 & 854.345 & 3296.39 & -1217.70 \\
\hline
\end{tabular}


TABLe 4: Numerical investigations of the $T(t), F(t)$, and $M(t)$ for Case 3 of the TFM model.

\begin{tabular}{|c|c|c|c|}
\hline \multirow{2}{*}{$t$} & \multicolumn{3}{|c|}{ Case 3} \\
\hline & $T(t)$ & $F(t)$ & $M(t)$ \\
\hline 0 & 0.9999 & 0.0001 & 0 \\
\hline 0.4 & 0.91790 & 0.08917 & -0.32811 \\
\hline 0.8 & 0.83361 & 0.11440 & -0.67315 \\
\hline 1.2 & 0.74964 & 0.07287 & -1.03638 \\
\hline 1.6 & 0.66871 & -0.03826 & -1.41954 \\
\hline 2 & 0.59367 & -0.22183 & -1.82483 \\
\hline 2.4 & 0.52746 & -0.48070 & -2.25501 \\
\hline 2.8 & 0.47317 & -0.81787 & -2.71345 \\
\hline 3.2 & 0.43398 & -1.23656 & -3.20419 \\
\hline 3.6 & 0.41323 & -1.74029 & -3.73200 \\
\hline 4 & 0.41439 & -2.33302 & -4.30250 \\
\hline 4.4 & 0.44111 & -3.01924 & -4.92219 \\
\hline 4.8 & 0.49724 & -3.80409 & -5.59860 \\
\hline 5.2 & 0.58682 & -4.69357 & -6.34036 \\
\hline 5.6 & 0.71418 & -5.69461 & -7.15737 \\
\hline 6 & 0.88394 & -6.81533 & -8.06087 \\
\hline 6.4 & 1.10104 & -8.06514 & -9.06365 \\
\hline 6.8 & 1.37086 & -9.45503 & -10.1801 \\
\hline 7.2 & 1.69921 & -10.9977 & -11.4268 \\
\hline 7.6 & 2.09246 & -12.7080 & -12.8221 \\
\hline 8 & 2.55758 & -14.6031 & -14.3867 \\
\hline 8.4 & 3.10226 & -16.7024 & -16.1442 \\
\hline 8.8 & 3.73500 & -19.0287 & -18.1207 \\
\hline 9.2 & 4.46520 & -21.6077 & -20.3460 \\
\hline 9.6 & 5.30331 & -24.4691 & -22.8529 \\
\hline 10 & 6.26098 & -27.6464 & -25.6788 \\
\hline
\end{tabular}

solve by using the DT method. The values are taken between 0 and 10 with the step size of 0.4 . These cases based on the above system of equations are written as follows:

Case 1. Taking the values of $\alpha=0.1, \beta=0.2, \delta=0.3$, $\gamma=0.4, d=0.5, I_{1}=0.9999, I_{2}=0.0001$, and $I_{3}=0$, model (1) takes the form as

$$
\begin{cases}\frac{\mathrm{d} T(t)}{\mathrm{d} t}=-0.1 F(t)-0.2, & T(0)=0.9999, \\ \frac{\mathrm{d} F(t)}{\mathrm{d} t}=0.3 T(t)+0.4 M(t), & F(0)=0.0001, \\ \frac{\mathrm{d} M(t)}{\mathrm{d} t}=-0.3 T(t)+0.2 M(t)-0.5, & M(0)=0 .\end{cases}
$$

The DT method is applied to solve the above model. By using the definitions of one-dimensional differential transformation and the corresponding transformation of equation (13), the obtained system becomes

$$
\begin{cases}(k+1) T(k+1)=-0.1 F(k)-0.2 \delta(k), & T(0)=0.9999, \\ (k+1) F(k+1)=0.3 T(k)+0.4 M(k), & F(0)=0.0001, \\ (k+1) M(k+1)=-0.3 T(k)+0.2 M(k)-0.5 \delta(k), & M(0)=0 .\end{cases}
$$

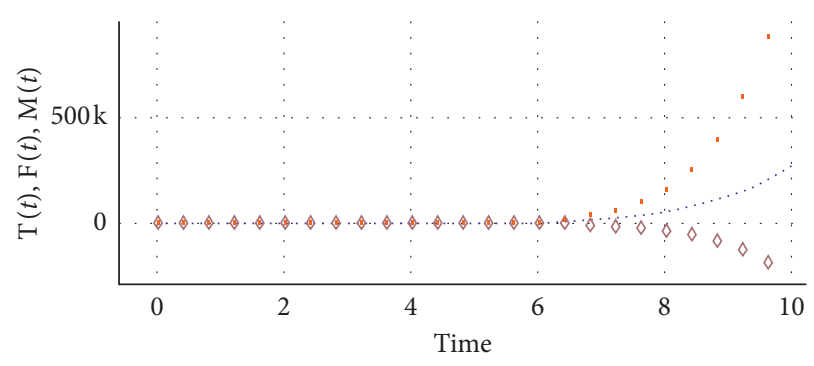

$$
\begin{array}{ll}
\ldots \mathrm{T}(t) \\
\Rightarrow \mathrm{F}(t) \\
\diamond \mathrm{M}(t)
\end{array}
$$

Figure 1: Nature of the $T(t), F(t)$, and $M(t)$ for Case 1 of the TFM model.

Case 2. Taking the values of $\alpha=-1, \beta=0.2, \delta=1, \gamma=0.4$, $d=0.1, I_{1}=0.9999, I_{2}=0.0001$, and $I_{3}=0$, model $(1)$ becomes

$$
\begin{cases}\frac{\mathrm{d} T(t)}{\mathrm{d} t}=F(t)-0.2, & T(0)=0.9999 \\ \frac{\mathrm{d} F(t)}{\mathrm{d} t}=T(t)+0.4 M(t), & F(0)=0.0001, \\ \frac{\mathrm{d} M(t)}{\mathrm{d} t}=-0.3 T(t)+0.2 M(t)-0.1, & M(0)=0 .\end{cases}
$$




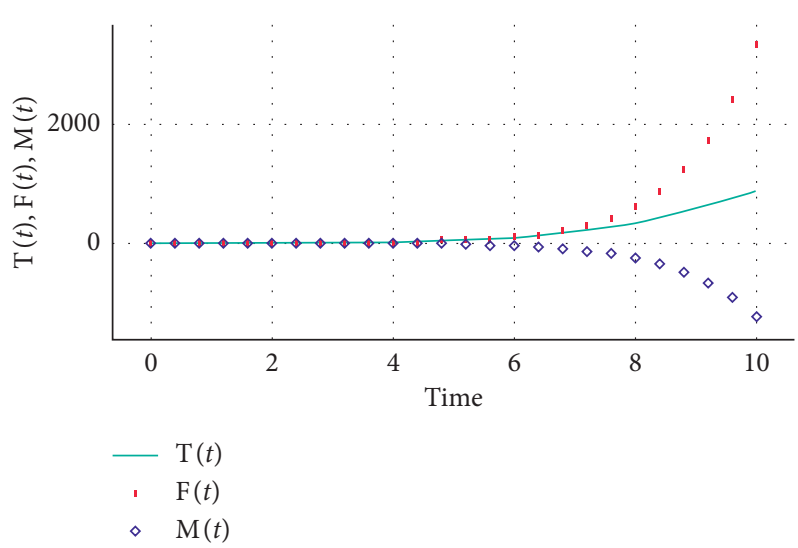

FIgure 2: Behavior of the $T(t), F(t)$, and $M(t)$ for Case 2 of the TFM model.

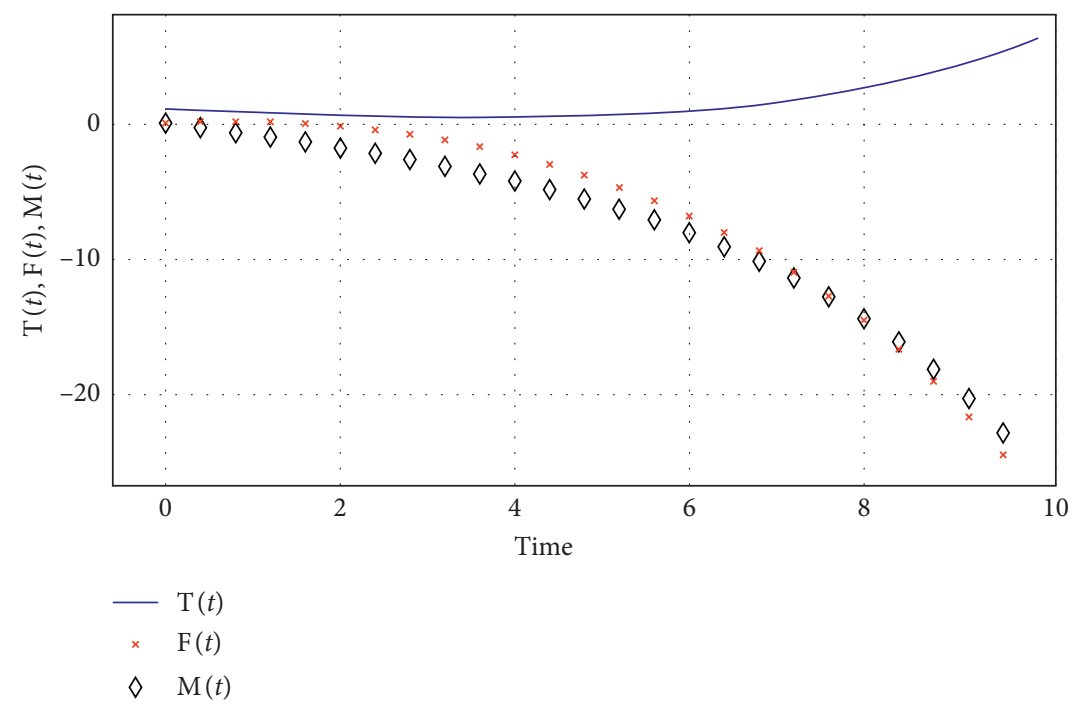

Figure 3: Behavior of the $T(t), F(t)$, and $M(t)$ for Case 3 of the TFM model.

The DT method is applied to solve the above equation (15). By using the definitions of one-dimensional differential transformation and the corresponding transformation of equation (15), the obtained system becomes

$$
\begin{cases}(k+1) T(k+1)=F(k)-0.2 \delta(k), & T(0)=0.9999, \\ (k+1) F(k+1)=T(k)+0.4 M(k), & F(0)=0.0001, \\ (k+1) M(k+1)=-0.3 T(k)+0.2 M(k)-0.1 \delta(k), & M(0)=0 .\end{cases}
$$

Case 3. Taking the values of $\alpha=2, \beta=0.2, \delta=2, \gamma=0.4$, $d=0.01, I_{1}=0.9999, I_{2}=0.0001$ and $I_{3}=0$, model (1) becomes

$$
\begin{cases}\frac{\mathrm{d} T(t)}{\mathrm{d} t}=-2 F(t)-0.2, & T(0)=0.9999 \\ \frac{\mathrm{d} F(t)}{\mathrm{d} t}=2 T(t)+0.4 M(t), & F(0)=0.0001, \\ \frac{\mathrm{d} M(t)}{\mathrm{d} t}=-0.3 T(t)+0.2 M(t)-0.01, & M(0)=0 .\end{cases}
$$

The DTM is applied to solve the above model. By using the definitions of one-dimensional differential transformation and the corresponding transformation of equation (17), the obtained system becomes

$$
\begin{cases}(k+1) T(k+1)=-2 F(k)-0.2 \delta(k), & T(0)=0.9999, \\ (k+1) F(k+1)=2 T(k)+0.4 M(k), & F(0)=0.0001, \\ (k+1) M(k+1)=-0.3 T(k)+0.2 M(k)-0.01 \delta(k), & M(0)=0 .\end{cases}
$$

Tables 2-4 show the different values of the $T(t), F(t)$, and $M(t)$ in the interval [0.10] with the step size of 0.4. The fixed initial values have been taken for all three cases. The 
numerical experimentations have been achieved by using the DT method. The details of each compartment for all the cases are provided in Tables 2-4. In Table 3, it is seen that by increasing the time, increment is seen in factors $T(t)$ and $F(t)$, while decrement is noticed in the values of $\mathrm{M}(t)$.

\section{Conclusion}

In the present study, a novel mathematical form of the nervous stomach model is introduced, which is based on three compartments namely tension, food, and medicine. The detail of each compartment is discussed and provided. The mathematical form of the TFM model is obtained in the form of three nonlinear differential equations, and numerical solutions have been tabulated and plotted in Tables $2-4$ and Figures $1-3$ by using a well-known differential transform scheme. The differential transformation scheme is recommended for all forms of differential equations due to the rapidity of its convergence and fewer computations. The considered method offers fast converging serial solutions, and furthermore, the accuracy of this solution can be improved by taking more terms in the solution. The differential transformation method is highly effective and produces approximate results of the model that have been investigated in this paper.

In the future, the novel nonlinear stomach model can be solved by using well-known artificial neural networks [24-33], and the differential transformation scheme can be applied by using fluid dynamics problems [34-36].

\section{Data Availability}

Our paper is not based on any data.

\section{Conflicts of Interest}

The authors declare that they have no conflicts of interest.

\section{Acknowledgments}

This work was partially supported by Ministerio de Ciencia, Innovaciony Universidades (grant number PGC2018-0971B-100) and Fundacion Seneca de la Region de Murcia (grant no. $20783 / \mathrm{PI} / 18)$.

\section{References}

[1] I. M. Modlin, From Prout to the Proton Pump, SchnetztorVerlag, Zeppelinstraße, Germany, 1995.

[2] W. Pagel, Joan Baptista Van Helmont: Reformer of Science and Medicine, Cambridge University Press, England, UK, 2002.

[3] N. Goodrick-Clarke, Paracelsus, vol. 1, North Atlantic Books, Berkeley, CA, USA, 1999.

[4] L. Spallanzani, Dissertazioni di Fisicaanimale e Vegetabile, Vol. 2, Presso La Societa’ Tipografica, Modena, Italy, 1780.

[5] R. F. De Réaumur, "Observations sur la digestion des oiseaux," Histoire de l'academieroyale des Sciences, vol. 266, p. $461,1752$.

[6] W. Prout, III. On the Nature of the Acid and Saline Matters Usually Existing in the Stomachs of Animals, vol. 114, pp. 45-49, Philosophical Transactions of the Royal Society of London, London, UK, 1824.

[7] W. Beaumont, "Further experiments on the case of Alexis san martin who was wounded in the stomach by a load of duckshot: detailed in the recorder for January 1825," 1825.

[8] H. H. Dale, "Comment on paper 23 in: adventures in physiology, a selection from the scientific publications of Sir Henry Hallett Dale," 1953.

[9] L. Popielski, " $\beta$-imidazolyläthylamin und die Organextrakte," Pflügers Archiv für die Gesamte Physiologie des Menschen und der Tiere, vol. 178, no. 1, pp. 214-236, 1920.

[10] W. M. Bayliss and E. Starling, "Preliminary communication on the causation of the so-called "peripheral reflex secretion" of the pancreas," The Lancet, vol. 159, no. 4099, p. 813, 1902.

[11] J. S. Edkins, "The chemical mechanism of gastric secretion 1," The Journal of Physiology, vol. 34, no. 1-2, pp. 133-144, 1906.

[12] J. W. Black, W. A. M. Duncan, C. J. Durant, C. R. Ganellin, and E. M. Parsons, "Definition and antagonism of histamine H2-receptors," Nature, vol. 236, no. 5347, pp. 385-390, 1972.

[13] J. R. Warren and B. Marshall, "Unidentified curved Bacilli on gastric epithelium in active chronic gastritis," The Lancet, vol. 321, no. 8336, pp. 1273-1275, 1983.

[14] W. Jaworski, Podrêcznikchoróbzoladka (Handbook of Gastric Diseases), Wydawnictwa Dziel Lekarskich Polskich, Warsaw, Poland, 1899.

[15] J. K. Zhou, Differential Transformation and Its Applications for Electrical Circuits, Huazhong University Press, Wuhan, China, 1986.

[16] D. Arslan, "The comparison study of the hybrid method with RDTM for solving Rosenau-Hyman equation," Applied Mathematics and Nonlinear Sciences, vol. 5, no. 1, pp. 267-274, 2020.

[17] H. Günerhan and E. Çelik, "Analytical and approximate solutions of fractional partial differential-algebraic equations," Applied Mathematics and Nonlinear Sciences, vol. 5, no. 1, pp. 109-120, 2020.

[18] Z. Sabir, H. Günerhan, and J. L. Guirao, "On a new model based on third-order nonlinear multisingular functional differential equations," Mathematical Problems in Engineering, vol. 2020, Article ID 1683961, 9 pages, 2020.

[19] M. Modanli and A. Akgül, "On solutions of fractional order telegraph partial differential equation by Crank-Nicholson finite difference method," Applied Mathematics and Nonlinear Sciences, vol. 5, no. 1, pp. 163-170, 2020.

[20] E. İlhan and İ.O. Kiymaz, "A generalization of truncated M-fractional derivative and applications to fractional differential equations," Applied Mathematics and Nonlinear Sciences, vol. 5, no. 1, pp. 171-188, 2020.

[21] M. S. M. Selvi and L. Rajendran, "Application of modified wavelet and homotopy perturbation methods to nonlinear oscillation problems," Applied Mathematics and Nonlinear Sciences, vol. 4, no. 2, pp. 351-364, 2019.

[22] W. Adel and Z. Sabir, "Solving a new design of nonlinear second-order Lane-Emden pantograph delay differential model via Bernoulli collocation method," The European Physical Journal Plus, vol. 135, no. 6, p. 427, 2020.

[23] D. Ziane, M. H. Cherif, C. Cattani, and K. Belghaba, "Yanglaplace decomposition method for nonlinear system of local fractional partial differential equations," Applied Mathematics and Nonlinear Sciences, vol. 4, no. 2, pp. 489-502, 2019.

[24] Z. Sabir, M. A. Z. Raja, M. Umar, and M. Shoaib, "Design of neuro-swarming-based heuristics to solve the third-order nonlinear multisingular Emden-Fowler equation," The European Physical Journal Plus, vol. 135, no. 6, p. 410, 2020. 
[25] Z. Sabir, F. Amin, D. Pohl, and J. L. Guirao, "Intelligence computing approach for solving second order system of the Emden-Fowler model," Journal of Intelligent \& Fuzzy Systems, pp. 1-16, 2020.

[26] Z. Sabir, H. A. Wahab, M. Umar, and F. Erdoğan, "Stochastic numerical approach for solving second order nonlinear singular functional differential equation," Applied Mathematics and Computation, vol. 363, Article ID 124605, 2019.

[27] Z. Sabir, H. A. Wahab, M. Umar, M. G. Sakar, and M. A. Z. Raja, "Novel design of the Morlet wavelet neural network for solving second order Lane-Emden equation," Mathematics and Computers in Simulation, vol. 172, pp. 1-14, 2020.

[28] M. Umar, Z. Sabir, and M. A. Z. Raja, "Intelligent computing for numerical treatment of nonlinear prey-predator models," Applied Soft Computing, vol. 80, pp. 506-524, 2019.

[29] M. A. Z. Raja, M. Umar, Z. Sabir, J. A. Khan, and D. Baleanu, "A new stochastic computing paradigm for the dynamics of nonlinear singular heat conduction model of the human head," The European Physical Journal Plus, vol. 133, no. 9, p. 364, 2018.

[30] Z. Sabir, M. A. Manzar, M. A. Z. Raja, M. Sheraz, and A. M. Wazwaz, "Neuro-heuristics for nonlinear singular Thomas-Fermi systems," Applied Soft Computing, vol. 65, pp. 152-169, 2018.

[31] M. A. Z. Raja, J. Mehmood, Z. Sabir, A. K. Nasab, and M. A. Manzar, "Numerical solution of doubly singular nonlinear systems using neural networks-based integrated intelligent computing," Neural Computing and Applications, vol. 31, no. 3, pp. 793-812, 2019.

[32] M. A. Z. Raja, Z. Sabir, N. Mehmood, E. S. Al-Aidarous, and J. A. Khan, "Design of stochastic solvers based on genetic algorithms for solving nonlinear equations," Neural Computing and Applications, vol. 26, no. 1, pp. 1-23, 2015.

[33] Z. Sabir and M. A. Z. Raja, "Numeric treatment of nonlinear second order multipoint boundary value problems using ANN, Gas, and the sequential quadratic programming technique," International Journal of Industrial Engineering Computations, vol. 5, no. 3, pp. 431-442, 2014.

[34] H. A. Wahab, Z. Zhiyu, M. Shoaib et al., "Numerical treatment for the three-dimensional Eyrin-Powell fluid flow over a stretching sheet with velocity slip and activation energy," Advances in Mathematical Physics, vol. 2019, Article ID 9860471, 12 pages, 2019.

[35] Z. Sabir, R. Akhtar, Z. Zhiyu et al., "A computational analysis of two-phase Casson nanofluid passing a stretching sheet using chemical reactions and gyrotactic microorganisms," Mathematical Problems in Engineering, vol. 2019, Article ID 1490571, 12 pages, 2019.

[36] M. Umar, Z. Sabir, A. Imran, A. Wahab, M. Shoaib, and M. Raja, "Three-dimensional flow of Casson nanofluid over a stretched sheet with chemical reactions, velocity slip, thermal radiation, and Brownian motion," Thermal Science, vol. 24, no. 5, pp. 2929-2939, 2020. 